\title{
Application of synchronized phasor measurements in RPA devices of distribution networks
}

\author{
Sergey A. Piskunov ${ }^{1, *}$, Alexey V. Mokeev ${ }^{1,2}$, and Evgeniy I. Khromtsov ${ }^{1,2}$ \\ ${ }^{1}$ Engineering Centre "Energoservice", Kotlasskaya st. 26, Arkhangelsk, Russia \\ ${ }^{2}$ Northern (Arctic) Federal University, Electroenergetics and Electrotechnics Department, Severnaya Dvina emb.17, Arkhangelsk, Russia
}

\begin{abstract}
The paper discusses the efficiency of RPA devices application with support of synchronized phasor measurements (SPM) at the step-down substations and in $6-20 \mathrm{kV}$ distribution networks. The main part of the paper presents a description of the algorithm developed by the authors for determining the damaged cable line during single-phase ground faults (SGF) in $6-20 \mathrm{kV}$ distribution networks with compensated and isolated neutral. The proposed algorithm makes it possible to realize reliable, fast-acting, and selective SGF protection in distribution cable networks acting either on a signal or on a trip. Based on the SGF protection using SPM, an SGF localization system can be created. This makes it easier to find a damaged cable line in the distribution network. The report presents the conditions for the application of this algorithm, which ensure the stability and reliability of its operation under various parameters of the network. The paper authors propose options for improving the SGF protection algorithm, which allows expanding the field of its application. The results of virtual modeling confirm the effectiveness of SGF protection based on the SPM using the developed algorithm. The final part of the paper contains examples demonstrating other areas of SPM application for RPA devices of step-down transformers, substations, cable distribution networks.
\end{abstract}

\section{Introduction}

The technology of synchronized phasor measurements is constantly expanding its field of application in the electric power industry. The use of SPM in distribution networks is considered promising, in particular, for the implementation of relay protection and automation functions [1-3]. Using SPM allows improving the technical excellence of relay protection devices, in some cases reducing the cost of their implementation in distribution networks.

One of the most common damages in 6-20 kV distribution networks is single-phase ground fault (SGF) [4]. At the same time, various neutral grounding methods are used in such networks, which complicates the task of developing unified SGF protection. Currently, there are many options for SGF protection for distribution networks [4-6]. But, there is no universal method that ensures the operation of SGF protection under various neutral grounding modes in the network and different types of SGF. Therefore, the development of new algorithms for determining the damaged section of the distribution network in SGF is a promising area for scientific research.

The use of zero-sequence current and voltage (ZSC, ZSV) SPM and modern ZSC sensors opens up new possibilities for the development of SGF protection in 6$20 \mathrm{kV}$ distribution networks [7-9]. Implementing the
WAMPAC principles of SPM-based systems improves SGF protection in various neutral grounding modes.

In addition to SGF protection, the use of SPM allows implementing fast-acting and selective protection of substation busbars, step-down transformers in $6-20 \mathrm{kV}$ distribution networks [10-12].

Consider in more detail the solution to the problem of implementing SGF protection in $6-20 \mathrm{kV}$ cable networks with isolated and compensated neutral.

\section{SPM application in SGF protection for distribution networks}

\subsection{General information}

The absence of current and voltage measuring transformers at most $10 \quad(6) / 0.4 \quad \mathrm{kV}$ transformer substations of urban cable distribution networks significantly complicates the search for a damaged cable line during single-phase ground faults. At the same time, the implementation of the SGF protection system in these networks will be justified only at relatively low costs for equipment, installation, and commissioning, as well as for maintenance of the SGF protection system.

An effective solution for determining a damaged cable line is related to the use of information about zerosequence current (ZSC) synchrophasors on network connections and zero-sequence voltage (ZSV)

\footnotetext{
* Corresponding author: s.piskunov@ens.ru
} 
synchrophasors at power centers: a step-down substation (SDS) or a distribution point (DP) [7-9].

This paper discusses the improvement of the above method of finding a damaged line in single-phase ground fault in distribution networks with isolated or compensated neutral.

\subsection{Operation principle of SGF protection}

SGF protection in 6-10 $\mathrm{kV}$ distribution networks based on the SPM can be implemented as a system for localizing a damaged section (cable line) with an action on the signal. A better system is based on disconnecting a damaged line and then automatically restoring the network [8].

To create the SGF localization system in the distribution network, localization sections are formed, among which a damaged cable line is searched in SGF. The section can be either a separate cable line or several cable lines having a direct connection and one power source.

To ensure the operation of the SGF localization system, devices for measuring ZSC synchrophasors are installed at each section at its ends. The boundaries of the SGF location search are determined by a pair of installed ZSC synchrophasor measuring devices. Thus, either a section of the cable network or a separate cable line can be located. The selection of sections is carried out based on the analysis of technical and economic indicators of the SGF localization system variants.

The SGF localization, in the general case, is carried out in the following order:

1. measurement of ZSC synchrophasors in all sections of the network and ZSV synchrophasors in sections of the power source center (SDS/DP);

2. calculation of technical parameters of each network section based on ZSC synchrophasors;

3. determination of the SGF location according to the above parameters.

The steady-state values of the ZSC synchrophasors for the network sections are presented in a complex form: the ZSC synchrophasor for the beginning of the network section $-\dot{I}_{0 n}^{b}$, for the end of the network section - $\dot{I}_{0 n}^{e}$. The index $n$ denotes the network section number in the system. Complex ZSC values in an exponential form: $\quad \dot{I}_{0 n}^{b}=I_{0 n}^{b} e^{j \varphi_{n}^{b}}, \dot{I}_{0 n}^{e}=I_{0 n}^{e} e^{j \varphi_{n}^{e}}$. The phase shift between ZSC synchrophasors at the beginning and the end of the section is determined by the expression:

$$
\Delta \varphi_{n}=\varphi_{n}^{b}-\varphi_{n}^{e}
$$

The phase shift $\Delta \varphi_{n}$ for the intact network section is defined by the size of his active and capacitive conductivities depending on parameters of the cable line and a ratio of the network sections conductivities.

The leakage current of a cable network section is usually capacitive since the capacitive conductivity of the intact cable is much greater than the active $Y_{C} \gg Y_{R}$. Therefore in the majority of calculations, we can consider that the leakage current of the intact network section has only a capacitive current component $\dot{I}_{c}$.

The algorithm for determining the SGF location using SPM is based on the directional current SGF protection principle [5] with an increase in its sensitivity and selectivity due to accurate measurements of the ZSC phase angle. High SPM resolution and accuracy allows to determinate the damaged network section, including in the network with compensation of capacitive current.

In general, the ZSC synchrophasors of the network sections in external SGF have one direction and are equally located relative to the base phasor (zerosequence voltage synchrophasor $\dot{U}_{0}$ ).

If one of the network sections with isolated or resistive-grounded neutral is damaged, the ZSC synchrophasors of the damaged network section will be directed opposite, i.e. the phase shift $\Delta \varphi$ between the synchrophasors $\dot{I}_{0 n}^{b}$ and $\dot{I}_{0 n}^{e}$ will be close to $180^{\circ}$. In addition, the module of one of the ZSC synchrophasors of the damaged network section will be the largest in the system. This makes it possible to use the traditional principles of ZSC directional protection [1] to detect a damaged section during SGF.

For a network with capacitive current compensation, it is impossible to determine the damaged section by the value $\dot{I}_{0 n}^{b}$ and $\dot{I}_{0 n}^{e}$ based on the current principle [4]. Therefore, only the zero-sequence active current, which is a few percent of the leakage current [6], can be used to determine the damaged section in the compensated network. For proper operation of the algorithm, a sufficiently high angle sensitivity is required, and ZSC sensors must have a small angular error. The use of ZSC and ZSV synchrophasors measuring devices in combination with the modern split-core ZSC sensors makes it possible to implement the directional principle of SGF determination in a network with compensated neutral.

The SGF current in the network with compensated neutral is determined by the active resistance of the arc suppression reactor coil (ASR) and the active conductivity of the network. Thus, the phase shift $\Delta \varphi$ for the damaged network section will be determined by the SGF current, the conductivity of the network section, and the conductivity of the entire network.

In general, if the capacitive conductivity of all network sections is equal, the maximum $\Delta \varphi$ will correspond to the damaged network section. In real conditions, the conductivity of sections can differ several times. At the same time, for short cable lines, with a low capacitance current, the phase shift $\Delta \varphi$ will vary within greater limits than for long cable lines with a large capacity due to the influence of the ZSC active component.

Therefore, the ratio of each section capacity to the capacity of the entire system should also be taken into account when determining a damaged area in a compensated neutral network. However, since the exact capacitance values of each section are usually unknown and are determined by reference data with a certain accuracy, the easiest way to take into account the above 
ratio is to use the measured ZSC values for the network section.

The proposed principle is described by the following expression:

$$
\psi_{\Delta n}=\Delta \varphi_{n} \frac{I_{0 n \max }}{I_{0 b}}
$$

where $\psi_{\Delta n}$ - angular characteristic of the network section with the number $n ; \Delta \varphi_{n}$ - phase shift of the section $n$; $I_{0 \text { max }}$ - maximum ZSC of the section $n ; \quad I_{0 b}$ - base current (in the simplest case $I_{0 b}=1 \mathrm{~A}$ ).

For network sections with small capacitive conductivity $I_{0 \text { nmax }}$ has a small value but at the same time size $\Delta \varphi_{n}$ increases. For sections with big capacitive conductivity $I_{0 \text { max }}$ increases, but $\Delta \varphi_{n}$ decreases. Thus, the angular characteristic $\psi_{\Delta n}$ allows reducing the influence of the network conductivity parameters on the determination of the damaged section during SGF.

By the maximum value of the angular characteristic $\psi_{\Delta}$ a damaged section of the network is determined. The proposed approach is also applicable for the network with isolated and resistive-grounded neutral.

At external SGF, the phase shift $\psi_{\Delta}$ shall not exceed the following value:

$$
\psi_{\Delta \min }=K_{r e s} \Delta \varphi_{\min } \frac{I_{C m i n}}{I_{0 b}},
$$

where $\Delta \varphi_{\min }-$ minimum threshold by phase shift determined by ZSC measurement error; $I_{\text {Cmin }}-$ minimum capacitance current among all network sections; $K_{\text {res }}$ - coefficient, which takes into account the measurement error of ZSC synchrophasors, as well as other possible errors of measurements and methodological calculations;

$I_{0 b}$ - base current.

\subsection{Requirements for measurement error and network parameters}

When determining $\Delta \varphi_{n}$ it is necessary to consider a measurement error of ZSC synchrophasors, which is defined by a sensor current error and accuracy of time synchronization. The current error of the used current sensors (LPCT) in the operating range makes 3\%, the maximum angular makes $2^{\circ}$. Thus, for pair of sensors is accepted $\Delta \varphi_{\min }=4^{\circ}$.

Since the algorithm operation in the network with compensated neutral depends on the capacitive component of the ZSC each section, as well as on their relationship with each other, it should be determined under what conditions the operation of the SGF localization system can be violated.

Consider an example of a network section with two cable lines between transformer substations (TS), as shown in Fig. 1.

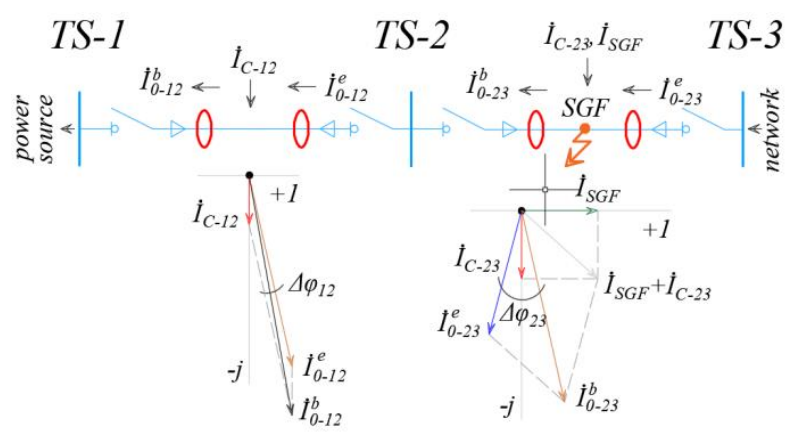

Fig. 1. Network section scheme and ZSC phasor diagram.

Suppose that the capacitive component of the ground fault current is fully compensated by the inductive current of the ASR coil. Then the SGF current $\dot{I}_{S G F}$ will be active. In turn, we suppose that the leakage current of network sections $\dot{I}_{C}$ is only capacitive. Fig. 1 shows two sections of the network - 12 and 23. For each, ZSC at the beginning and end of the line at SGF $\left(\dot{I}_{0}^{b}\right.$ and $\left.\dot{I}_{0}^{e}\right)$ are indicated.

Suppose that SGF is on section 23 (call it damaged). The ZSC at the beginning of section 23 is then defined by:

$$
\dot{I}_{0-23}^{b}=\dot{I}_{0-23}^{e}+\dot{I}_{C-23}+\dot{I}_{S G F},
$$

where $\dot{I}_{0-23}^{e}-$ ZSC at the end of section $23 ; \dot{I}_{C-23}-$ leakage current of section $23 ; \dot{I}_{S G F}$ - SGF current.

The ZSC synchrophasor argument at the end of section 23 is defined from the expression:

$$
\varphi_{23}^{e}=-\operatorname{arctg}\left(\frac{I_{0-23 C}^{e}}{I_{0-23 A}^{e}}\right)
$$

where $I_{0-23 A}^{e}-$ active ZSC component at the end of the section $23 ; I_{0-23 C}^{e}-$ capacitive ZSC component at the end of the section 23 .

The ZSC synchrophasor argument at the beginning of section 23 is defined from the expression:

$$
\varphi_{23}^{b}=-\operatorname{arctg}\left(\frac{I_{0-23 C}^{e}+I_{C-23}}{-I_{0-23 A^{+} I_{S G F}}^{e}}\right) .
$$

The phase shift between ZSC at the beginning and end of section 23:

$$
\Delta \varphi_{23}=-\operatorname{arctg}\left(\frac{I_{0-23 C^{+I} C-23}^{e}}{-I_{0-23 A^{+}}^{e} S G F}\right)+\operatorname{arctg}\left(\frac{I_{0-23 C}^{e}}{I_{0-23 A}^{e}}\right)
$$

In the case when the ZSC at the end of section 23 is capacitive:

$$
\Delta \varphi_{23}=-\operatorname{arctg}\left(\frac{I_{0-23 C}^{e}+I_{C-23}}{I_{S G F}}\right)+90^{\circ} .
$$

Fig. 2 shows an example of calculating the phase shift for the damaged section 23 depending on different initial conditions. 


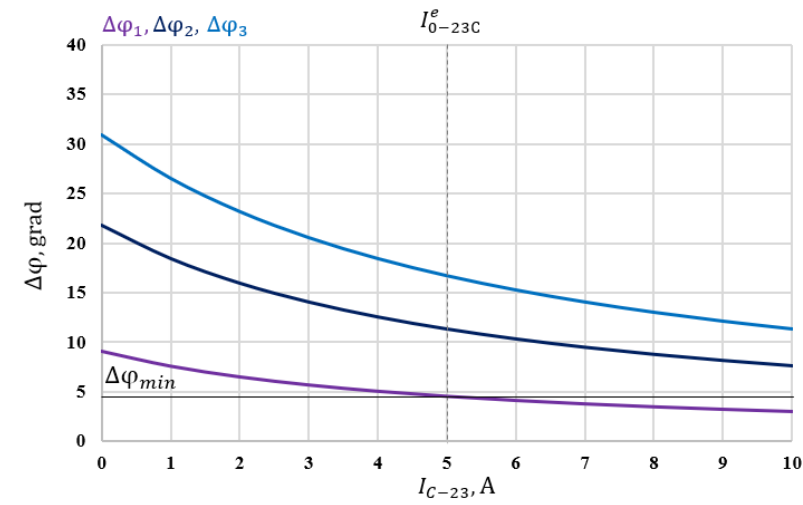

Fig. 2. Phase shift calculation for a damaged section.

Fig. 2 shows: $I_{0-23 C}^{e}$ - the capacitive ZSC component at the end of section 23 (equal to $5 \mathrm{~A}$ in this example), $\Delta \varphi_{\text {min }}$ - the minimum phase shift for normal operation of the algorithm taking into account possible measurement error, $\Delta \varphi_{1}, \Delta \varphi_{2}, \Delta \varphi_{3}$ - phase shift at SGF current of 1,2 , and $3 \mathrm{~A}$.

It can be seen from expression (6) that with full compensation of the active and capacitive SGF current component, the operation of the algorithm becomes impossible at the fundamental frequency of the ZSC. In real conditions, the active SGF current component in networks with compensated neutral is present and makes several percent of the capacitive current $[4,6]$. As calculations show, this is enough to perform a stable operation of the algorithm.

With different ratios $I_{C-23} / I_{0-23 C}^{e}$, characterizing the ratio of the section capacitive current to the capacitive current of the network rest, values $\Delta \varphi$ change. As shown in Fig. 2, the permissible values $\Delta \varphi$ at different values $I_{S G F}$ are obtained at the ratio $I_{C-23} / I_{0-23 C}^{e} \leq 0.5$, i.e., when the capacity of any network section does not exceed $50 \%$ of the other sections total capacity. At $I_{S G F}$ more than $2 \%$ of the capacitive network current, the algorithm will also work at large ratios $I_{C-23} / I_{0-23 C}^{e}$. The actual value of the SGF current is not known in advance, therefore, when selecting sections of the SGF localization system, the above ratio should be taken into account.

Consider calculating the phase shift for undamaged sections. The expression for calculating the phase shift for section 12 (Fig.2) will be as follows:

$$
\Delta \varphi_{12}=-\operatorname{arctg}\left(\frac{I_{0-12 C}^{e}+I_{C-12}}{I_{0-12 A}^{e}}\right)+\operatorname{arctg}\left(\frac{I_{0-12 C}^{e}}{I_{0-12 A}^{e}}\right)
$$

where $I_{0-12 A}^{e}$ - active ZSC component at the end of the section $12 ; I_{0-12 C}^{e}$ - capacitive ZSC component at the end of the section $12 ; \dot{I}_{C-12}$ - leakage current of section 12.

From the expression (9), it can be seen that for the supply substation (distribution point) connections that do not have a damaged section, the phase shift $\Delta \varphi$ at SGF will be small (in the absence of a leakage current active component will be zero) and will not exceed the value of the ZSC measurement angular error. Thus, such sections can easily be identified as intact.
The most difficult is the determination of the damaged connection section at low SGF currents and a large difference in the ratio of individual section's capacitive currents. Therefore, it is important to set acceptable limits on the applicability of the algorithm to avoid its incorrect operation.

Table 1 shows the research results on the selection of optimal operating algorithm conditions at different ratios $I_{C}$ connection sections. For these purposes, a mathematical model of a $10 \mathrm{kV}$ network with compensated neutral in the Simulink was tested under various conditions.

Table 1. Optimal ratios $I_{C} / I_{C}$ of damaged connection sections.

\begin{tabular}{|c|c|c|c|c|c|c|c|c|c|c|}
\hline \multirow{2}{*}{$I_{0}^{e}, \mathrm{~A}$} & \multicolumn{10}{|c|}{$I_{C}, \mathrm{~A}$} \\
\cline { 2 - 12 } & 1 & 2 & 3 & 4 & 5 & 6 & 7 & 8 & 9 & 10 \\
\hline 28.5 & 0.03 & 0.07 & 0.10 & 0.14 & 0.17 & 0.21 & 0.24 & 0.28 & 0.31 & 0.35 \\
\hline 14.3 & 0.07 & 0.14 & 0.21 & 0.28 & 0.35 & 0.42 & 0.49 & 0.56 & 0.63 & 0.70 \\
\hline 9.6 & 0.10 & 0.21 & 0.31 & 0.42 & 0.52 & 0.63 & 0.73 & 0.84 & 0.94 & 1.05 \\
\hline 7.2 & 0.14 & 0.28 & 0.42 & 0.56 & 0.70 & 0.84 & 0.97 & 1.11 & 1.25 & 1.39 \\
\hline 5.8 & 0.17 & 0.35 & 0.52 & 0.69 & 0.87 & 1.04 & 1.22 & 1.39 & 1.56 & 1.74 \\
\hline 4.8 & 0.21 & 0.42 & 0.62 & 0.83 & 1.04 & 1.25 & 1.46 & 1.66 & 1.87 & 2.08 \\
\hline 4.1 & 0.24 & 0.48 & 0.73 & 0.97 & 1.21 & 1.45 & 1.69 & 1.94 & 2.18 & 2.42 \\
\hline 3.6 & 0.28 & 0.55 & 0.83 & 1.10 & 1.38 & 1.65 & 1.93 & 2.21 & 2.48 & 2.76 \\
\hline 3.2 & 0.31 & 0.62 & 0.93 & 1.24 & 1.55 & 1.85 & 2.16 & 2.47 & 2.78 & 3.09 \\
\hline 2.9 & 0.34 & 0.68 & 1.03 & 1.37 & 1.71 & 2.05 & 2.39 & 2.74 & 3.08 & 3.42 \\
\hline
\end{tabular}

In Table $1, I_{C \Sigma}$ is the total capacitive connection current. The left column shows the ZSC values at the end of the intact section, which is connected to the damaged section $\left(I_{0}^{e}\right)$, and the upper row shows the values of the capacitive current of this section $\left(I_{C}\right)$. For each case values $\psi_{\Delta}$ were determined for the damaged and intact sections at different values of the SGF current and the active network conductivity. Green highlights the relationships in which the $\psi_{\Delta}$ of the damaged section was maximized in the network with a certain safety factor, i.e. the damaged section can be determined uniquely in this connection.

The selected range of $I_{0}^{e}$ and $I_{C}$ can be expanded, but from the ratios established in Table 1 , it is obvious that the capacitive current of any section should not exceed $85-90 \%$ of the entire connection capacitive current. Otherwise, the algorithm can correctly determine the connection with the damaged section, but it is wrong to localize the SGF section in this connection.

Thus, two main conditions are formed for the selection of SGF sections according to the proposed algorithm:

1. the capacitance current of any section shall not exceed $50 \%$ of the entire network total capacitance current;

2. the capacitive current of the connection's section shall not exceed $90 \%$ of the connection's total capacitive current. 
These algorithm limits can be extended by introducing an additional condition for determining the angular characteristic:

$$
\psi_{\Delta n}=\Delta \varphi_{n} \frac{I_{0 n \max }}{I_{C \Sigma}},
$$

where: $\Delta \varphi_{n}$ - phase shift of the section $n ; \quad I_{0 n \max }-$ maximum ZSC of the section $n ; I_{C \Sigma}$ - total capacitive connection current up to and including the section from the side opposite the power source.

The introduction of an angular characteristic (10) will make it possible to abandon the above conditions of the algorithm. At the same time, the exact definition of $I_{C \Sigma}$ is not always possible, in addition, the network scheme can be changed during operation. Therefore, the application of the characteristic (10) is recommended for cases where the conditions of the algorithm operation are not met.

The presented principle of algorithm operation implies not only certain requirements for the accuracy of ZSC measurements but also the correct orientation of current sensors when they are installed on TS/DP. Incorrect orientation of the current sensors in any of the sections may lead to incorrect algorithm operation.

\subsection{Modeling of protection operation}

Consider an example of the algorithm operation on a simple network model with compensated neutral, shown in Fig. 3.

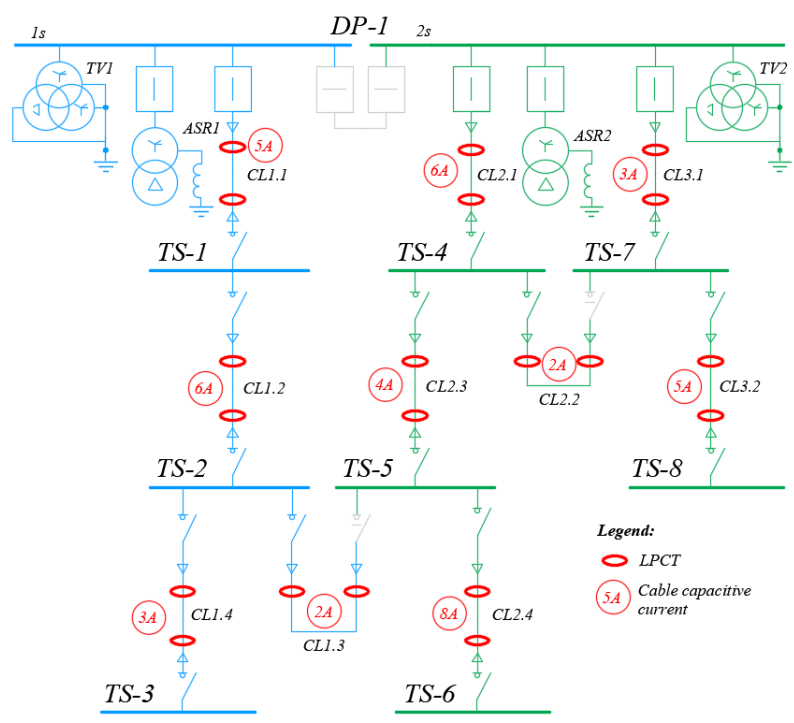

Fig. 3. Compensated neutral network model.

The $10 \mathrm{kV}$ network is powered by a DP-1 on which two bus sections (highlighted in color) are separated in normal operation. DP-1 has three outgoing connections. Each cable connection line is numbered (1.1-1.4, 2.1-2.4, 3.1-3.2). Connections 1 and 2 are located on the first section DP-1, 3 - on the second section. Fig. 3 shows the LPCT installation places, as well as the calculated values of each cable line capacitive currents.

The operating conditions of the algorithm proposed above are observed for the scheme in Fig.3, but the limit ratio of capacitive currents for cable line 1.2 is selected: $(3+2) / 6=0.83$.

For connections having large length cable lines at the end (with high capacitance), the second condition is met for almost any network circuit. But for connections that have at the beginning long cable lines with a large capacitive current, and at the end very short, it is necessary to use the expression (10) to improve the algorithm operation.

The arc suppression reactors ASR-1 and ASR-2 are tuned to fully compensate for the capacitance current of sections 1 and 2. Active resistance of ASR coils is set within $5 \%$ of their inductive resistance.

The following is the calculation of the network sections angular characteristics for each case of SGF - on all cable lines and at external SGF.

The calculation is made in the Simulink virtual model. The results are shown in Fig. 4 and in Table 2.

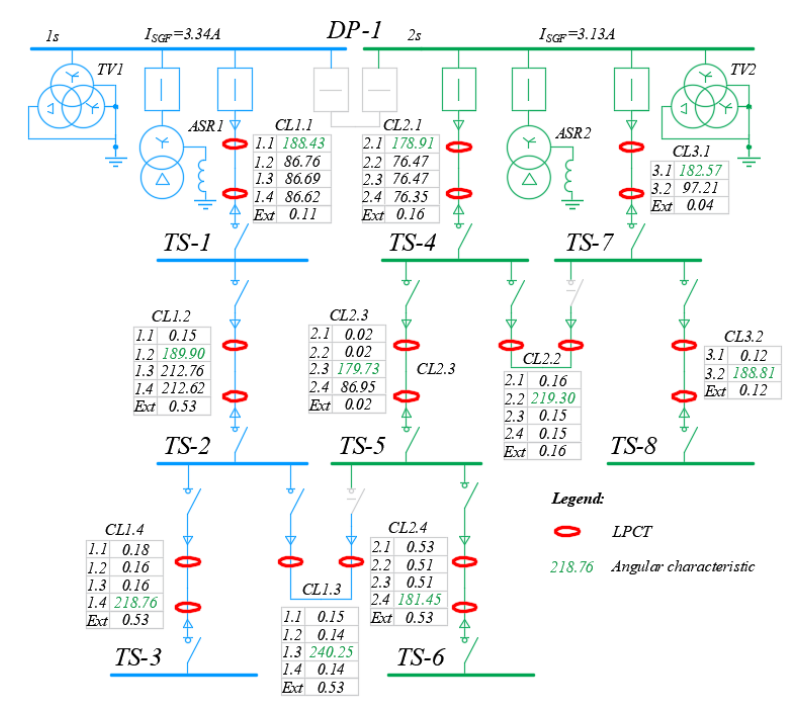

Fig. 4. Simulink calculation results.

Table 2. Results of $\psi_{\Delta}$ calculation for network sections.

\begin{tabular}{|c|c|c|c|c|c|c|c|c|}
\hline \multirow{2}{*}{$\begin{array}{c}\text { SGF } \\
\text { location }\end{array}$} & \multicolumn{8}{|c|}{ Network section } \\
\hline & 1.1 & 1.2 & 1.3 & 1.4 & 2.1 & 2.2 & 2.3 & 2.4 \\
\hline 1.1 & 188.4 & 0.2 & 0.2 & 0.2 & - & - & - & - \\
\hline 1.2 & 86.8 & 189.9 & 0.1 & 0.2 & - & - & - & - \\
\hline 1.3 & 86.7 & 212.8 & 240.3 & 0.2 & - & - & - & - \\
\hline 1.4 & 86.6 & 212.6 & 0.1 & 218.7 & - & - & - & - \\
\hline 2.1 & - & - & - & - & 178.9 & 0.2 & 0.0 & 0.5 \\
\hline 2.2 & - & - & - & - & 76.5 & 219.3 & 0.0 & 0.5 \\
\hline 2.3 & - & - & - & - & 76.5 & 0.1 & 179.7 & 0.5 \\
\hline 2.4 & - & - & - & - & 76.4 & 0.1 & 86.9 & 181.5 \\
\hline $\begin{array}{c}\text { External } \\
\text { SGF }\end{array}$ & 0.1 & 0.5 & 0.5 & 0.5 & 0.2 & 0.2 & 0.0 & 0.5 \\
\hline
\end{tabular}

The calculation results confirm the effectiveness of the algorithm. At the same time, for sections 1.3 and 1.4, similar $\psi_{\Delta}$ values are obtained for the selected ratio of capacitive currents $(0.83)$.

Table 3 shows the calculation results of $\psi_{\Delta}$ by expression (10). 
Table 3. Results of $\psi_{\Delta}$ calculation by expression (10).

\begin{tabular}{|c|c|c|c|c|c|c|c|c|}
\hline \multirow{2}{*}{$\begin{array}{c}\text { SGF } \\
\text { location }\end{array}$} & \multicolumn{8}{|c|}{ Network section } \\
\cline { 2 - 10 } & 1.1 & 1.2 & 1.3 & 1.4 & 2.1 & 2.2 & 2.3 & 2.4 \\
\hline 1.1 & 11.7 & 0.0 & 0.1 & 0.1 & - & - & - & - \\
\hline 1.2 & 5.4 & 17.3 & 0.1 & 0.1 & - & - & - & - \\
\hline 1.3 & 5.4 & 19.3 & 120.1 & 0.1 & - & - & - & - \\
\hline 1.4 & 5.4 & 19.3 & 0.1 & 72.9 & - & - & - & - \\
\hline 2.1 & - & - & - & - & 8.9 & 0.1 & 0.0 & 0.1 \\
\hline 2.2 & - & - & - & - & 3.8 & 109.7 & 0.0 & 0.1 \\
\hline 2.3 & - & - & - & - & 3.8 & 0.1 & 14.9 & 0.1 \\
\hline 2.4 & - & - & - & - & 3.8 & 0.1 & 7.3 & 22.7 \\
\hline $\begin{array}{c}\text { External } \\
\text { SGF }\end{array}$ & 0.0 & 0.1 & 0.3 & 0.2 & 0.0 & 0.1 & 0.0 & 0.1 \\
\hline
\end{tabular}

The calculation results of $\psi_{\Delta}$ according to expression (10) show that implementation with conditions 1 and 2 is not required in this case. All values of maximum angular characteristics for each SGF location are obtained with a large safety factor, which provides high efficiency of the algorithm. But at the same time, additional data was used to form the algorithm, which in some cases (for example, when changing the network operation scheme) may be different. Thus, the choice of expression (2) or (10) depends on the specific initial operating conditions of the network.

Check correctness of algorithm operation at external SGF. As per expression (3), the minimum threshold of actuation by angular characteristic is calculated. The minimum capacitance current $I_{C m i n}$ corresponds to sections 1.3 and $2.2(2 \mathrm{~A})$. Take the factor $K_{\text {res }}=1.5$. Then by expression (3), we get:

$$
\psi_{\Delta \min }=K_{r e s} \Delta \varphi_{\min } \frac{I_{C \min }}{I_{0 b}}=1.5 \cdot 4 \cdot 2=12 .
$$

The maximum obtained $\psi_{\Delta}$ value at external SGF is 0.53 . Thus, the condition is satisfied.

\subsection{Implementation}

The SGF localization subsystem is implemented as part of the Digital RES software and hardware complex [8].

The SGF localization subsystem includes:

- electromagnetic split-core ZSC sensor (LPCT);

- device for measurement of ZSC and ZSV synchrophasors (ENLZ);

- device ENCM-3 for data collection and transmission with built-in GLONASS/GPS receiver and built-in modem $3 \mathrm{G} / 2 \mathrm{G}$;

- server with SCADA software and ES-Graph software.

For the operation of the system for determining the emergency network section during SGF, it is additionally necessary to measure the $3 U_{o}$ voltage in phasor form similar to currents $3 I_{o}$. The number of voltage measurements $3 U_{o}$ is equal to the number of bus sections of all power source centers of the distribution network. Measurement of $3 U_{o}$ can be carried out at both SDS and DP equipped with voltage measuring transformers with winding $3 U_{o}$ (open triangle).

For the measurement of ZSC and ZSV synchrophasors, the classic SPM algorithm is implemented in the ENLZ devices [13]. A specially designed averaging FIR filter [12] is used.

The Digital RES complex as a pilot project was implemented in the Arkhangelsk city cable networks. Currently, the Digital RES software and hardware complex is installed and is in trial operation on a number of TS and DP of the MUP «Electroset» in Cherepovets.

\subsection{Improving SGF protection}

The algorithm can accelerate its operation and increase its efficiency by using the values of ZSC synchrophasors in the transient process of SGF. However, it should be borne in mind that many conditions of the network operation in the transition mode differ significantly from the steady-state SGF mode. Therefore, it is necessary to check the stability of the algorithm in a transient process.

At the moment of a ground fault, the ASR inductance practically does not affect the transient process of the capacitive current component. Thus, the ZSC distribution in a network with compensated neutral at the time of transition is much the same as the ZSC distribution in a network with isolated neutral, which allows using the principle of directed protection to determine a damaged section.

The problem of algorithm implementation for localizing the damaged section in the transient process of SGF based on SPM is the short-term duration of this transient process in cable networks, as well as its wave nature [3], which leads to an increase in the influence of the LPCT measurement error on the operation of the algorithm.

Fig. 5 shows the graph of the relative ZSC and ZSV values in section 2.1 at the SGF in section 2.3 for the network scheme, refer to Fig. 3.

As can be seen from Fig. 5, the ZSC and ZSV in the transient process contain higher harmonic components, which can be observed in a stable SGF, depending on the parameters of the network and equipment. In this case, the phase angle of the fundamental ZSC harmonic synchrophasors can vary widely. In addition, if the ZSC exceeds the limit multiplicity of the LPCT sensor, it can be saturated.

Fig.6 shows an example of the angular characteristics $\psi_{\Delta}$ calculation for the transient process at SGF in section 1.4 .

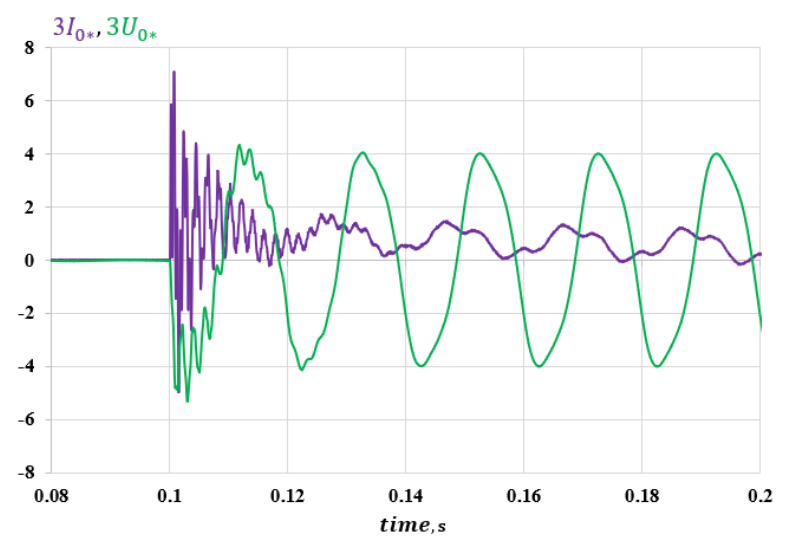

Fig. 5. The transition process of a stable SGF. 


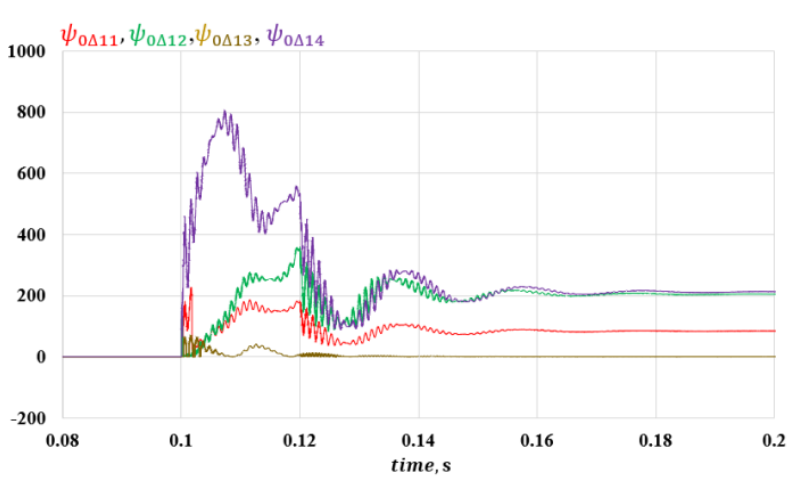

Fig. 6. Angular characteristics calculation for the transient process at SGF in section 1.4.

As can be seen from Fig.6, the angular characteristics of sections 1.2 and 1.4 are close in the steady-state SGF mode, as was shown in Table 2. However, in the transition process, the angular characteristic on the damaged segment 1.4 is significantly greater than the angular characteristic 1.2.

The Simulink modeling results confirm that almost always in the first periods of the transition, the angular characteristic of the damaged network section calculated according to expression (2) is significantly greater than the angular characteristics of other sections. At the same time, the effect of the transition process on the LPCT and other equipment operation should also be taken into account in practice. Therefore, the implementation issue of the SGF localization system based on the SPM using the data of the SGF transition process requires further research.

In addition to the above version of SGF protection based on SPM, other algorithms based on synchronized measurements of ZSC and ZSV can be proposed in the network with compensated neutral.

The perspective direction is the measurement of ZSC higher harmonic synchrophasors [7]. This principle assumes that higher harmonics are contained in the ZSC and ZSV during SGF, and their level is high enough to accurately measure them. Some studies and developments confirm the possibility of determining the SGF direction by the level of ZSC higher harmonics [14$15]$.

Harmonic components appear in the SGF current during the transient process due to the fault current loop parameters. The other part of the higher harmonics currents remains in the stable SGF current. They appear due to the presence of harmonic components in generators EMF, due to saturation of the power transformers magnetic core, and the presence of uneven and asymmetric loads (rectifiers, electric traction, melting furnaces, etc.) [3].

The ground fault current is most often dominated by the fifth and seventh harmonic components. As a result of resonant phenomena, the eleventh and thirteenth harmonics can appear in the network during the burning of the grounding arc [3].

The main problem of the SGF localization algorithm implementation on the principle of absolute measurement of ZSC higher harmonics in the network with compensated neutral is a non-constant level of harmonics depending on the network configuration, distance from the power source, presence of asymmetric load, etc. [8].

Fig. 7 shows an example of $\psi_{\Delta}$ calculation based on the 3rd ZSC harmonic for the scheme according to Fig.3. In the Simulink calculation model, an additional source of higher harmonics, whose power is less than $1 \%$ of the main source, was added to implement the method. Thus, this source simulates the presence of higher harmonics from non-linear elements (power transformers, motor load, rectifiers, etc.).

Damaged section - cable line 1.4. As can be seen from Fig. 7, the algorithm operation when measuring ZSC higher harmonics is similar to its operation on the fundamental ZSC harmonic in a network with an isolated neutral - oppositely directed ZSC synchrophasors occur in a damaged line, which allows it to be easily identified.

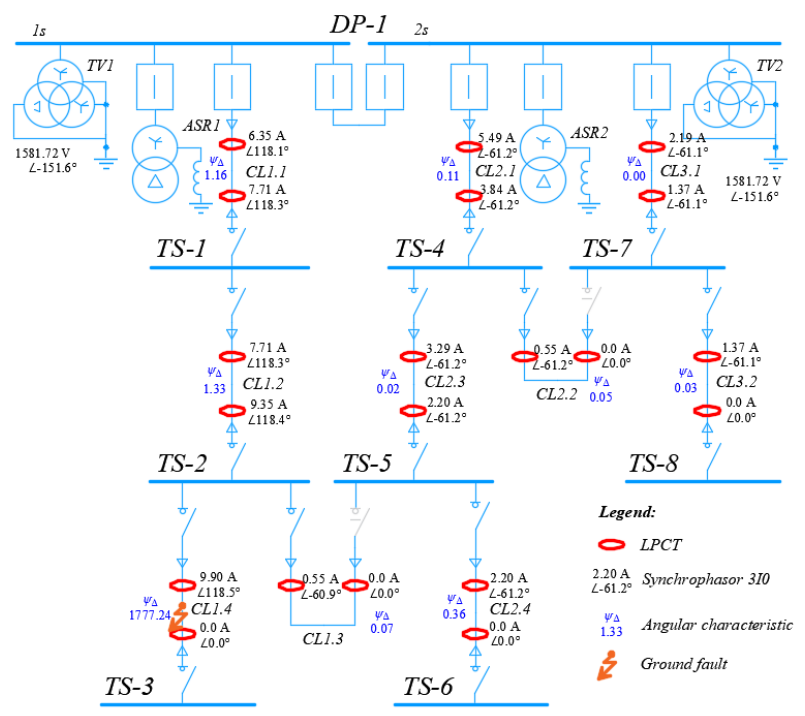

Fig. 7. Example of $\psi_{\Delta}$ calculation for $3^{\text {rd }}$ ZSC synchrophasors.

To implement such an algorithm, studies of the real level of harmonics in the ZSC and ZSV in various networks with compensated neutral during SGF are required. In addition, the ZSC sensors used must also provide a sufficiently accurate measurement of the higher harmonic synchrophasors.

\section{SPM application for distribution network RPA}

Taking advantage of digital substation technologies and synchronized phasor measurements will allow realizing efficient relay protection devices against phase-to-phase short circuits $[10,11]$.

Due to the exchange of synchrophasors and GOOSE messages between the protections at the substation, it is possible to implement inherently selective protections. For example, in the substation input relay protection device, by using the information on the synchrophasors of medium voltage connection current and voltage synchrophasors on the substation buses, it is possible to implement bus inherently selective protection $[10,11]$. 
Based on the use of current and voltage synchrophasors in combination with fast communications at the digital substation, it will become possible to apply more widely the distance and differential principles of protection using additional signs of alarm recognition and the implementation of inherently selective protection based on information exchange between protections.

\section{Conclusions}

Operation of SGF localization system in 6-10 kV distribution cable network based on SPM is considered. The used SGF localization algorithm depending on the neutral grounding mode is described in detail. Limits and conditions of algorithm applicability are indicated, examples of its use are considered. Calculations were made in the Simulink virtual model. The results of the experiments were confirmed on the real model of the cable network, as well as during pilot operation. Alternative versions of using SPM for SGF localization are proposed.

The prospect of using SPM technology for relay protection of SDS and DP is associated with the possibility of wide implementation of fast-acting inherently selective protections.

\section{References}

1. Integrating Synchrophasor Technology into Power System Protection Applications (NASPI, 2016)

2. Wide area protection \& Control technologies (CIGRE, WG B5.14, Paris, 2016)

3. Synchrophasor Monitoring for Distribution Systems: Technical Foundations and Applications (NASPI, 2018)

4. V.A. Shuin, A.V. Gusenkov, Ground fault protection in electrical networks 6-10 kV (M, 2001)

5. M.A. Shabad, Protection against single-phase ground faults in 6-35 kV networks (SPb, 2007)

6. F.A. Likhachev, Ground faults in networks with isolated neutral and with capacitive current compensation (M, 1971)

7. V. Bovykin, et all, CIGRE B5 Colloquium in Tromsø, PS1-122 (2019)

8. A.I. Popov, et al., E3S Web of Conferences, 216, 01045 (2020)

9. K.V. Petrov, et all, IEEE International Conference RPA (2020)

10. A.V. Mokeev, E.I. Khromtsov, Relay protection and automation, 4 (2019)

11. A.V. Mokeev et all, E3S Web of Conferences 216, 01044 (2020)

12. A.V. Mokeev, IEEE International Conference SIBCON, 17082680 (2017)
13. IEEE/IEC International Standard 60255-118-12018, Part 118-1 (2018)

14. V.A. Shuin, et all, International Conference Methodological issues of reliability research of large energy systems, Irkutsk, 71 (1) (2020)

15. T.U. Vinokurova, et all, Vestnik IGEU, Ivanovo, 3 (2015) 